\title{
BMJ Open WHO recommendations on physical activity versus compliance rate within a specific urban population as assessed through IPAQ survey: a cross-sectional cohort study
}

\author{
Pawel Macek, ${ }^{1}$ Malgorzata Terek-Derszniak, ${ }^{2}$ Marek Zak, ${ }^{3}$ Malgorzata Biskup,,${ }^{2,3}$ \\ Przemyslaw Ciepiela, ${ }^{4}$ Halina Krol, ${ }^{3}$ Jolanta Smok-Kalwat, ${ }^{5}$ Stanislaw Gozdz ${ }^{3,5}$
}

To cite: Macek P, TerekDerszniak M, Zak M, et al. WHO recommendations on physical activity versus compliance rate within a specific urban population as assessed through IPAQ survey: a cross-sectional cohort study. BMJ Open 2019;9:e028334. doi:10.1136/ bmjopen-2018-028334

\section{- Prepublication history for} this paper is available online. To view these files, please visit the journal online (http://dx.doi. org/10.1136/bmjopen-2018028334).

Received 3 December 2018 Revised 24 April 2019

Accepted 26 April 2019

Check for updates

(c) Author(s) (or their employer(s)) 2019. Re-use permitted under CC BY-NC. No commercial re-use. See rights and permissions. Published by BMJ.

${ }^{1}$ Department of Epidemiology and Cancer Control, Holycross Cancer Centre, Kielce, Poland ${ }^{2}$ Department of Rehabilitation, Holycross Cancer Centre, Kielce, Poland

${ }^{3}$ Faculty of Medicine and Health Sciences, The Jan Kochanowski University, Kielce, Poland

${ }^{4}$ Clinic of Oncological Surgery, Holycross Cancer Centre, Kielce, Poland

${ }^{5}$ Clinic of Clinical Oncology, Holycross Cancer Centre, Kielce, Poland

Correspondence to

Dr Marek Zak; mzak1@onet.eu

\section{ABSTRACT}

Objective The study was designed to address the following three key areas, that is, (1) evaluate overall level of physical activity in the residents of a mid-sized, Central-European city, (2) compliance level with WHO's recommendations on physical activity in leisure time and (3) actual impact of select socioeconomic factors on the physical activity level within the study population. Methods Assessment of the source data collected for 4619 participants (1532 men and 3087 women, aged 4565 years; mean age $56.41 \pm 5.31$ years) was completed. Three levels of physical activity, and compliance level with pertinent WHO recommendations was evaluated, based on International Physical Activity Questionnaire (long form). Multilevel logistic regression models of socioeconomic factors associated with moderate-level, high-level physical activity, and WHO recommendations were developed. Results Data analyses revealed that $6.19 \%$ of the study participants $(n=286)$ engaged in low-level physical activity, $48.86 \%$-in moderate-level activity, while high-level activity was reported in $44.94 \%$ of them. Compliance with pertinent WHO recommendations was higher in men aged 44-55 years, boasting upper-level education, living without a partner and in the persons with a net income over $€ 1140$ per household.

Conclusions Overall level of physical activity in the residents of a mid-sized, Central-European city was established as moderate. Pertinent WHO recommendations on physical activity were met by $4.2 \%$ of the subjects only.

\section{INTRODUCTION}

Lack of physical activity in conjunction with sedentary lifestyle is deemed by far the most hazardous to any modern-day population. ${ }^{1-6}$ If physical activity is to offer any beneficial effects whatsoever, certain key criteria must be complied with, for example, frequency and intensity. Physical activity should be approached comprehensively in terms of actual exertion in different areas of everyday life, that is, at work, at home, while travelling
Strengths and limitations of this study

- Polish-Norwegian Study is one of the first cohort research projects in Central and Eastern Europe focused on the lifestyle-related, chronic disease risk factors.

- Assessment of health-promoting, social paradigms helps identify prevalent perception of health issues, depending on the respondents' specific positioning within a social structure.

- Failure to pursue a health-promoting lifestyle by the study participants was often owed not so much to individual ill will, as to an interplay of several environmental factors characteristic for a specific socioecological paradigm.

- Even though the recruitment process, based on voluntary participation, never affected the intrinsic value of the study outcomes, due caution is still recommended in their interpretation.

- No objective assessment methods (eg, accelerometer, pedometer) were employed in the study protocol.

or commuting and in leisure time. ${ }^{1}$ Assessment of physical activity level is instrumental in making viable predictions on individual health status. It also plays an essential role in promoting one's physical and mental wellbeing, as well as public health at large. ${ }^{2}$

Ongoing evolution of individual lifestyles is invariably affected by ongoing changes within human environment. Overall deficiency in physical activity, especially when associated with a diversity of modern conveniences commonly available in everyday life, holds serious implications for human health status. Lack of regular physical activity contributes to the development of chronic diseases, for example, cardiovascular diseases, metabolic disorders, cancer, respiratory diseases, musculoskeletal disorders and neurological diseases. $^{478}$ 
Physical activity is vital for retaining good health, ${ }^{9-12}$ shaping a health-promoting lifestyle, as well as offers an attractive option for spending leisure time. Lack of physical activity in a population may contribute to placing a significant burden on both national economy and public healthcare system, primarily by way of appreciably increasing expenditure on the healthcare services for the individuals unfit to work. Much wider appreciation of an essential role physical activity plays in prevention and treatment of assorted lifestyle diseases has generated increased interest in the physically active dimension of life.

Even though beneficial effects of physical activity are widely acknowledged, $>30 \%$ of adults in Europe seldom get involved in any physical exercise regimens. ${ }^{13}$ According to WHO, any physical activity is better than none at all, with the benefits largely independent of sex, race or ethnicity. ${ }^{14}{ }^{15}$ WHO recommends that healthy adults aged 18-64 years should engage in moderate-intensity physical activity for at least $150 \mathrm{~min} /$ week, or high-intensity activity for $75 \mathrm{~min} /$ week, whereas for extra health benefits, an individual should engage in at least $300 \mathrm{~min}$ of moderate-intensity activity, or $150 \mathrm{~min}$ of high-intensity activity per week, or their combined equivalent. The recommended minimal time of physical activity is 10 min. ${ }^{16}$ According to long International Physical Activity Questionnaire (IPAQ) form, physical activity of a particular study population is assessed in four domains (work, home, transport, free time). The minimum duration of a single physical activity is set at $10 \mathrm{~min}$, at the very least. Any such an activity is comprised of physical activities originating in different domains, lasting at least 10 min each. Should an adult be unable to undertake such an activity with the health reasons in mind, WHO recommends undertaking physical activity at any viable level whatsoever. ${ }^{1617}$

The present study aimed to present the physical activity of the Polish-Norwegian Study (PONS) participants by way of addressing three key areas:

1. Evaluation of overall level of physical activity in the participants of a mid-sized, Central-European city.

2. Compliance level with WHO's recommendations on physical activity in leisure time.

3. Actual impact of select socioeconomic factors on the level of physical activity within the study population.

\section{METHODS}

\section{Participants and procedures}

Relevant data of 4619 study participants were subjected to verification. The project aimed to collect comprehensively structured data on essential health and well-being factors, as well as gain some insights into the causes of morbidity and mortality within the population under study. The PONS Project 'Establishment of infrastructure for population health research in Poland', based on collaboration between Polish and Norwegian scientists, aimed to collect extensive data in the population under study on the key factors regarding individual health status and well-being, as well as gain some insights into the actual causes of morbidity and mortality in Poland. The PONS survey was conducted in the city of Kielce (The Świętokrzyskie Region). The study population was comprised of persons aged 45-64 years. This project was in fact a continuation of the international Health Evolution Monitoring (HEM)-Closing the Gap project, pursued in the Oncology Centre, Warsaw.

Recruitment to the PONS study was based on voluntary participation, supported by a broad media campaign. Men and women aged 45-64 years, who were permanent residents at the time of recruitment (110 000 eligible persons), were invited to participate in the study. They were recruited from the general populations of two geographically distinct regions, that is, urban district - city of Kielce (60 000 residents aged 45-64 years, of whom 8000 (13\%) were included in the PONS population sample), and one rural district- Kielce region (50 000 residents aged 45-64 years, including $5000(10 \%)$ in the PONS population sample). The study sites were selected in due consideration of acknowledged risk patterns for major chronic diseases, essential exposure factors, economic development level, relative stability of the population, adequate local infrastructure, and longterm local commitment to the Project's objectives.

The study protocol comprised the following components: Health Status Questionnaire, medical examinations, anthropometric measurements (body weight, height, waist circumference, hip circumference) and collection of biological material (urine and blood samples). The questionnaire covered the following sections: health status (general health status, disease history), demographic and social factors (gender, age, education, marital status, professional work, type of occupation, total monthly net income of all household members), mental health and lifestyle (smoking, alcohol consumption, diet, physical activity). Information on gender, education, marital status, professional work and total monthly net income of all household members was collected through a direct interview.

Based on the evaluation of the completeness and coherence of data (both horizontal and vertical) pertaining to select socioeconomic factors, that is, gender, age, education, marital status, employment, job type, net monthly income per household and the self-admitted level of physical activity, as assessed by the long IPAQ form, the data for 181 participants were excluded from further assessment. The data collected for 58 participants were found deficient to a considerable extent. The age of two participants failed to meet the inclusion criteria. The data collected for the remaining 121 participants were removed, in line with the IPAQ methodology (eg, total duration of physical activity should not exceed $960 \mathrm{~min} /$ day). Detailed analyses were carried out for 4619 participants that took part in the study protocol (1532 men and 3087 women, aged 45-65 years; mean age of $56.41 \pm 5.31$ years). The study group characteristics are presented in table 1 . 
Table 1 Study group characteristics in consideration of PA levels, and compliance with WHO recommendations

\begin{tabular}{|c|c|c|c|c|}
\hline & $n=4619$ & Moderate-level PA & High-level PA & WHO recommendations \\
\hline Variable & $\%$ & n (\%) & n (\%) & n (\%) \\
\hline \multicolumn{5}{|l|}{ Sex } \\
\hline Male & 33.17 & $661(43.15)$ & 726 (47.39) & $90(5.87)$ \\
\hline \multicolumn{5}{|l|}{ Age group (years) } \\
\hline $56-65$ & 58.67 & $1398(51.59)$ & $1136(41.92)$ & $73(2.69)$ \\
\hline \multicolumn{5}{|l|}{ Education } \\
\hline $\begin{array}{l}\text { Lower level } \\
\text { (primary or vocational) }\end{array}$ & 14.48 & $295(44.10)$ & $325(48.58)$ & $12(1.79)$ \\
\hline $\begin{array}{l}\text { Upper level } \\
\text { (secondary or higher) }\end{array}$ & 85.52 & $1962(49.67)$ & $1751(44.33)$ & $182(4.61)$ \\
\hline In a relationship & 75.88 & $1716(48.96)$ & $1567(44.71)$ & $141(4.02)$ \\
\hline \multicolumn{5}{|l|}{ Professional activity } \\
\hline Professional active & 54.71 & $1079(42.70)$ & $1284(50.81)$ & $146(5.78)$ \\
\hline Professional inactive & 45.29 & $1178(56.31)$ & $792(37.86)$ & $48(2.29)$ \\
\hline \multicolumn{5}{|c|}{ Net income per household } \\
\hline$<€ 450$ & 11.82 & $275(50.37)$ & 235 (43.03) & $12(2.20)$ \\
\hline From $€ 450$ to $€ 679$ & 16.56 & $381(49.80)$ & $345(45.10)$ & $16(2.09)$ \\
\hline From $€ 680$ to 1139 & 20.09 & $463(49.89)$ & $408(43.97)$ & $40(4.31)$ \\
\hline
\end{tabular}

n, absolute number; PA, physical activity.

The IPAQ (long form) was the research tool of choice. Physical activity was evaluated in four areas of everyday life, that is, at a place of work, while commuting daily, while doing regular household chores, and during leisure time. As per the IPAQ methodology, the participants were divided according to their total physical activity levels, that is, low, moderate and high. ${ }^{18} 19$

- Low-level activity (individuals who do not meet the criteria for the other two categories, physical activity at a level <600 MET-min/week).

- Moderate-level activity (physical activity at a level of 600-1500 MET-min/week, or 1500-3000 MET-min/ week, although with 1 or 2 days comprising high-intensity exercise).

- High-level activity (1500 MET-min/week, although with at least 3 days comprising high-intensity exercise, over $3000 \mathrm{MET}-\mathrm{min} /$ week).

\section{Outcomes}

The primary and secondary outcomes of interest were the self-reported level of compliance with WHO recommendations on physical activity, and moderate-level and highlevel physical activity during leisure time, respectively.

\section{Confounders and mediators}

We made use of six self-reported potential confounders or mediator variables (ie, gender, age, level of education, marital status, occupational activity and net income per household). All variables were quantitative; their values fully grounded in the survey questionnaire applied in the study protocol.

\section{Missing data}

Net income per household variable contained $36.91 \%$ $(n=1705)$ of missing data. This might well be attributed to the participants' reluctance to have their income disclosed (eg, participants with low income could intentionally skip their low income, as they regarded this as a violation of their privacy). The probable missing not a random type of missing data was assumed. The missing data were construed as yet another value for the categorical variable 'net income per household', and labelled 'not specified'. In the following parts of the study, multilevel logistic regression models were developed, while making use of both the full data, inclusive of this brand-new category of missing data, and without them. 


\section{Analysis}

Multilevel logistic regression was applied. Six multilevel regression models were developed. Model 1 and 1a, model 2 and 2a, model 3 and 3 a presented socioeconomic factors associated with moderate-level, high-level physical activity and WHO recommendations on physical activity during leisure, respectively. Models with 'a' inserted into the name (eg, model 1a) were based on a portion of the data after the deletion of all cases $(n=1705)$ of missing data on the net income per household variable. Effects sizes were presented as ORs with 95\% CI. CIs were based on the profiled log-likelihood function. Akaike information criterion (AIC) was a measure of model adjustment. The best one of all the models tested was the one with the smallest AIC. All statistical analyses were completed using the R V.3.4.2.

\section{Patient and public involvement}

The authors represent that neither any patients, nor any members of the public were in any way involved in designing, nor in conducting the study protocol. In view of the actual specifics of its design, the authors do not envisage having the study outcomes disseminated to its participants.

\section{RESULTS}

Table 1 shows the characteristics of the study group. In total, 4619 subjects were assessed ( $66.83 \%$ women), with body mass index values ranging $16.51-52.28 \mathrm{~kg} / \mathrm{m}^{2}$ (mean $27.79 \pm 4.41 \mathrm{~kg} / \mathrm{m}^{2}$ ). It was established that $6.19 \%$ of the subjects engaged in low-level physical activity, $48.86 \%$ in moderate-level activity, while high-level activity was observed in $44.94 \%$ of them. Compliance with WHO recommendations on physical activity during leisure was observed in $4.21 \%$ of the individuals.

Pursuant to the results yielded by an unadjusted analysis (table 2), it was observed that female gender, group aged 56-65 years and the upper-level education were the categories associated with the increased odds of moderate-level physical activity. Professionally active status was associated with the decreased odds of moderate-level physical activity. The opposite pattern of socioeconomic categories was observed within the high-level physical activity. Male gender, group aged 45-55 years, upper-level education, professionally active status and a net income per household ranging $€ 680-€ 1.139$, and over, were associated with the increased odds of compliance with WHO recommendations on physical activity during leisure.

Table 3 shows ORs and 95\% CI of the socioeconomic factors for the six multilevel logistic regression models. Three are based on the full data $(n=4619)$, and another three are based on the partial data $(\mathrm{n}=2914)$, as explained in the Methods section. Following the adjustment for individual variables, it was investigated how various socioeconomic factors affected the likelihood that specific levels of physical activity under study would be encountered more frequently than others. Based on the models 1 and 2, the relevant categories of variables, that is, education, professional activity and a net income per household, were juxtaposed against the likelihood of moderate-level and high-level physical activity. Models 3 and $3 a$ were similar and fitted, based on the same predictors. Female gender, aged 56-65 years, living in a relationship, were associated with the decreased odds, whereas upper-level education, and a net income per household

Table 2 Unadjusted analysis of factors associated with PA levels, and compliance with WHO recommendations

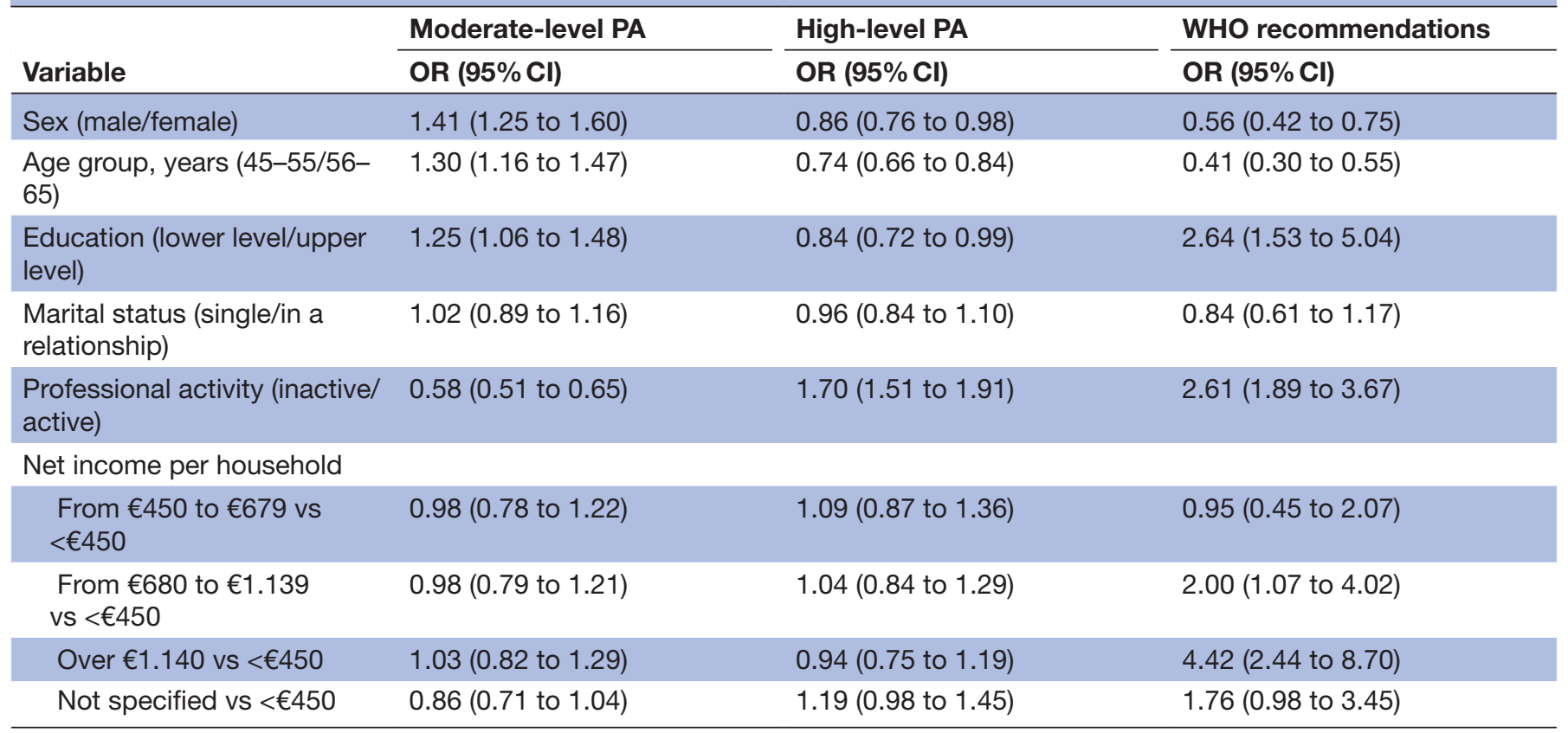

PA, physical activity. 


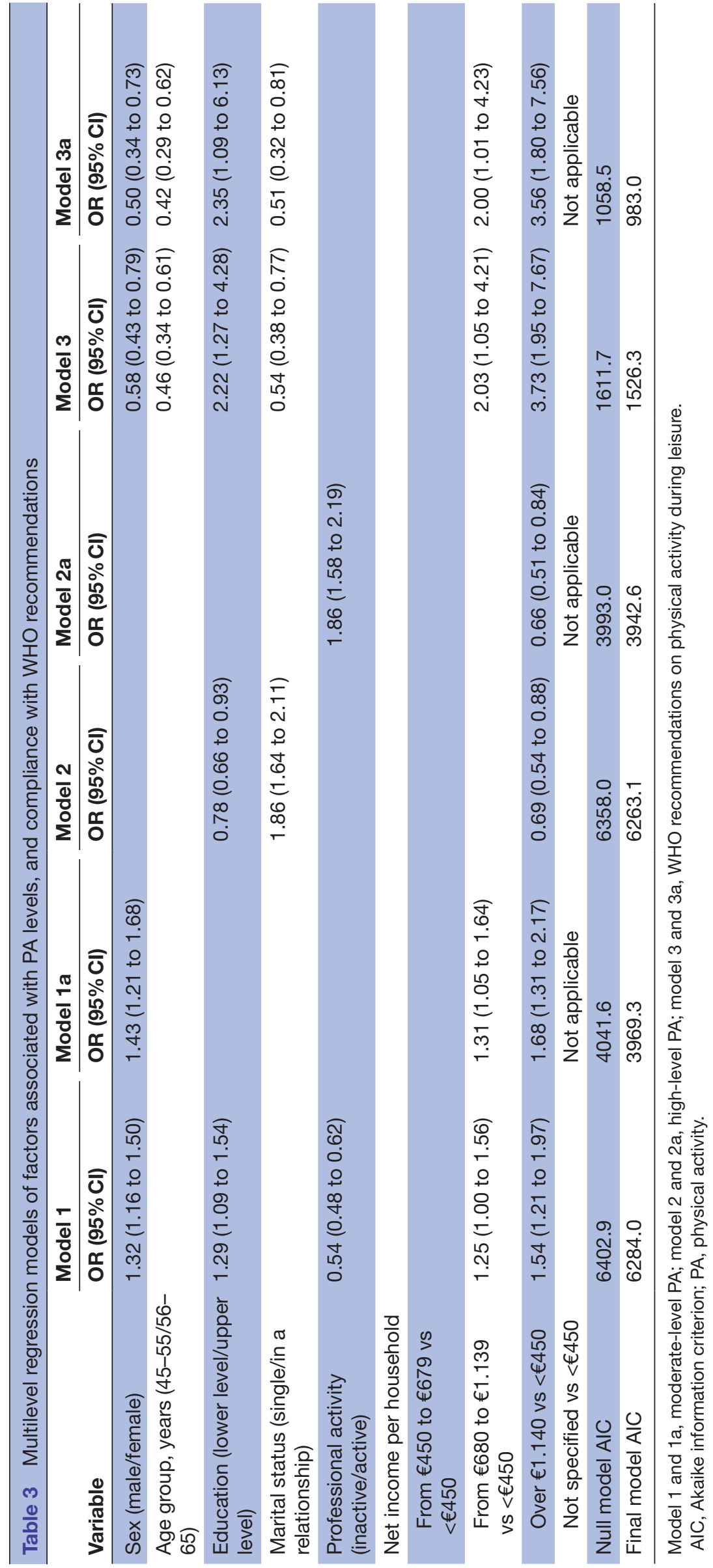

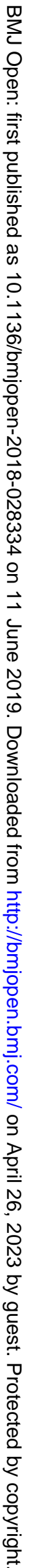


ranging $€ 680-€ 1.139$, and over, were associated with the increased odds of compliance with WHO recommendations on physical activity during leisure. For all the fitting models, the reduced AIC was noted, following the inclusion of individual variables.

\section{DISCUSSION}

Diverse and complex impact of sociodemographic factors on the level of physical activity pursued by the participants was assessed. High net income per household member increased the likelihood of moderate physical activity being undertaken, while reducing that of a high-level one. Net income also modified the likelihood of compliance with pertinent WHO recommendations. This impact was also clearly detectable with respect to the model taking into account the missing data as a variable within a separate category of net income per household member, as well as when disregarding it. In the present study, the implementation of WHO recommendations for physical activity in leisure time proved to be by far the most complex in terms of the actual impact of the sociodemographic factors under study. In the regression models, only the type of occupational activity had no impact on its occurrence. This only goes to highlight a great diversity of conditions that may modify basic manifestations of human endeavours within a lifetime; spontaneous physical activity in leisure time among them.

In other studies, a high level of physical activity was reported for the subjects living in New Zealand, Czech Republic, USA and Australia. On the other hand, the subjects in the countries, such as Belgium, Japan, Brazil and Taiwan, were the least likely to undertake highlevel physical activity. ${ }^{20}$ The situation in Finland is altogether different. Throughout over 30 years of research, a systematic increase in physical activity in leisure time was noted. ${ }^{21}$ This increase may well be attributable to systematically pursued promotion of physical activity throughout the country. Nevertheless, around one-third of adult population across the world fails to pursue individually any health-promoting physical activity. ${ }^{22}$

In order to monitor public health status effectively, it is essential to determine the number of individuals who undertake physical activity, and to recognise and understand which specific modifiable factors and motives influence an individual willingness to do so. ${ }^{23}{ }^{24}$ The most important finding of the present study consists in establishing the discrepancy between the subjects' level of self-admitted physical activity, and the actual implementation level of pertinent WHO recommendations. Even though well over $80 \%$ of the subjects was allocated into the moderate-level and high-level categories, specific WHO recommendations were met by $4.2 \%$ of them only.

The results of research on physical activity pursued within a population, as conducted in Europe, revealed that a majority of respondents failed to comply with WHO recommendations on physical activity. ${ }^{1825-31}$
Around one-quarter of the European population does not follow WHO recommendations on physical activity, which might be attributable to certain inequalities between respective countries, as well as to the ones encountered within them. ${ }^{32}$ Along with a diversity of ongoing changes in man's immediate environment, significant changes in people's lifestyle are simply inevitable. Civilisational progress accompanied by all-embracing automation and mechanisation in all major areas of life have appreciably contributed to overall reduction of people's physical activity. Research into physical activity has highlighted several factors that actually differentiate people's approach to this issue, for example, age, gender, individual health condition and individual motivation. ${ }^{33}$

Considering the differences in the level of physical activity pursued across the Western and Central and Eastern European countries, it seems prudent enough to have the programmes aimed at promoting individual physical activity designed and structured on a local level. Failure to pursue a health-promoting lifestyle by the study participants was often owed not so much to individual ill will, as to an interplay of several environmental factors characteristic for a specific socioecological paradigm. This approach would then stand a far better chance of actually reaching out to the least physically active population groups. Taking into account the differences in compliance with WHO specific recommendations on physical activity, it would also be advisable to consider whether these guidelines should actually be addressed to all social strata within the same scope. Current WHO recommendations take into account the age factor only. In the light of the latest research, it would seem rather prudent to assume that a number of other socioeconomic factors acknowledged to impact individual level of physical activity, for example, gender, education, type of occupation, economic status and region of residence, also be granted due consideration. Assessment of health-promoting paradigms helps identify prevalent perception of health issues, depending on the respondents' specific positioning within a social structure. Disregarding those other factors by WHO may well inadvertently become instrumental in the non-compliance with its recommendations on physical activity in respective European countries, especially across Central and Eastern Europe. About $40 \%$ of 53 European countries have never developed their own guidelines on physical activity in which the specific WHO recommendations would be reflected to some extent. This group comprises Poland and the countries of Central and Eastern Europe. ${ }^{34}$

Following their systemic transformation in the early 1990s, the countries of Central and Eastern Europe strive to match overall quality of life in Western Europe. As a result of embracing wholesale consumerism, physical activity as a lifestyle factor has been pushed to a much more inferior position in an order of life's priorities. Besides, state-of-the-art technological advances and brand-new social trends emerging in the developing countries make sedentary lifestyle steadily more and more 
common across Central and Eastern Europe. WHO indicates that hypokinesia and sedentary lifestyle are deemed legitimate risk factors for lifestyle diseases. ${ }^{35}$

With a view to appreciably increasing the chances for compliance with WHO recommendations on physical activity, overall public awareness of an appreciably advantageous effect of physical activity on individual health status should definitely be raised. Owing to social, cultural and economic differences encountered between Western and Eastern Europe, specific recommendations on physical activity should be developed on a local level.

It is rather hard to determine whether the WHO recommendations are obsolete, or whether the countries of Central and Eastern Europe have not as yet reached that particular stage of development whereupon their implementation has become a standard lifestyle requirement. In order to take up this challenge, it is vital to establish the actual level of physical activity and the nature of the relationship between physical activity and specific socioeconomic factors within a particular locality, and consequently have the recommendations on physical activity effectively adapted to the specific needs of a local population.

Further research is required, with a view to identifying potential environmental factors specific to a particular region/locality, which could account for the differences in population behavioural models, especially in relation to physical activity across Europe at large.

\section{Study limitations}

One of the more obvious drawbacks was that the study protocol did not provide for any verification of an individual physical activity through objective assessment methods (eg, accelerometer, pedometer). Even though the recruitment process, based on voluntary participation, never affected the intrinsic value of the study outcomes, due caution is still recommended in their interpretation. As the study design did not envisage any follow-up, either, there was no opportunity to establish whether the very fact of addressing the survey questionnaire by the respondents may have in any way affected their individual lifestyles, for example, encourage them to take up any type of regular physical activity.

\section{CONCLUSION}

The results yielded by the present study indicate that despite undertaking a moderate level of physical activity, the residents of a mid-sized, Central-European city, that is, study participants failed to comply with WHO recommendations on physical activity in leisure time. The level of physical activity and compliance with WHO recommendations were determined by socioeconomic factors, for example, gender, age, education, marital status and the net monthly income per household. The effect of each of these factors, however, was by no means discrete; they either complemented or eliminated one another.
Individual factors may affect overall physical activity level either favourably or adversely.

Contributors PM: conceptualisation, formal analysis, investigation, methodology, project administration, resources, supervision, writing — review and editing. MT-D: data curation, formal analysis, project administration, resources, software, validation, writing —original draft. MZ: conceptualisation, formal analysis, investigation, methodology, validation, writing—original draft, writing—review and editing. MB: software, funding acquisition, supervision and resources. PC: funding acquisition, software, supervision and visualisation. HK and JS-K: data curation, project administration and resources. SG: formal analysis, funding acquisition, investigation, supervision, validation, visualisation, writing —original draft. All coauthors read and approved the final version of the manuscript.

Funding Implementation of a research project (Ref. No PNRF-228-Al-1/07: PONSPolish-Norwegian Study) 'Establishment of the infrastructure to facilitate studies on the health status of Poland's population': PNRF-228-Al-1/07, financed out of the Polish-Norwegian Foundation Research Fund. The project is supported under the programme established by the Minister of Science and Higher Education, named 'Regional Initiative of Excellence', spanning the period 2019-2022; Project No 024/ RID/2018/19, amount of financing: PLN 11999000.00

Competing interests None declared.

Patient consent for publication Not required.

Ethics approval The present study was duly approved by a local Ethics Review Committee, Faculty of Health Sciences (Approval Ref. No. 25/2015), The Jan Kochanowski University (JKU), Kielce, Poland.

Provenance and peer review Not commissioned; externally peer reviewed.

Data sharing statement The datasets generated during and/or analysed during the current study are available from the corresponding author on reasonable request.

Open access This is an open access article distributed in accordance with the Creative Commons Attribution Non Commercial (CC BY-NC 4.0) license, which permits others to distribute, remix, adapt, build upon this work non-commercially, and license their derivative works on different terms, provided the original work is properly cited, appropriate credit is given, any changes made indicated, and the use is non-commercial. See: http://creativecommons.org/licenses/by-nc/4.0/.

\section{REFERENCES}

1. Uijtdewilligen L, Yin JD, van der Ploeg HP, et al. Correlates of occupational, leisure and total sitting time in working adults: results from the Singapore multi-ethnic cohort. Int J Behav Nutr Phys Act 2017:14:169.

2. Liu Y, Wen W, Gao YT, et al. Level of moderate-intensity leisure-time physical activity and reduced mortality in middle-aged and elderly Chinese. J Epidemiol Community Health 2018;72:13-20.

3. Stringhini S, Carmeli C, Jokela M, et al. Socioeconomic status, non-communicable disease risk factors, and walking speed in older adults: multi-cohort population based study. BMJ 2018;360:k1046.

4. Skillgate E, Pico-Espinosa OJ, Hallqvist J, et al. Healthy lifestyle behavior and risk of long duration troublesome neck pain or low back pain among men and women: results from the Stockholm Public Health Cohort. Clin Epidemiol 2017;9:491-500.

5. Lee IM, Shiroma EJ, Lobelo F, et al. Effect of physical inactivity on major non-communicable diseases worldwide: an analysis of burden of disease and life expectancy. Lancet 2012;380:219-29.

6. Fernandez-Navarro P, Aragones MT, Ley V. Leisure-time physical activity and prevalence of non-communicable pathologies and prescription medication in Spain. PLoS One 2018;13:e0191542.

7. van Zutphen M, Kampman E, Giovannucci EL, et al. Lifestyle after Colorectal Cancer Diagnosis in Relation to Survival and Recurrence: A Review of the Literature. Curr Colorectal Cancer Rep 2017; 13:370-401.

8. Kruk J. Health and economic costs of physical inactivity. Asian Pac J Cancer Prev 2014;15:7499-503.

9. Lopez RP, Hynes HP. Obesity, physical activity, and the urban environment: public health research needs. Environ Health 2006;5:25.

10. Wojciechowska P. Physical activity and human health. Med Stud Med 2015;30:254-60.

11. Pan SY, Cameron C, Desmeules M, et al. Individual, social, environmental, and physical environmental correlates with physical 
activity among Canadians: a cross-sectional study. BMC Public Health 2009;9:21.

12. White J, Greene G, Kivimaki M, et al. Association between changes in lifestyle and all-cause mortality: the Health and Lifestyle Survey. $J$ Epidemiol Community Health 2018;72:711-4.

13. Marques A, Sarmento H, Martins J, et al. Prevalence of physical activity in European adults - Compliance with the World Health Organization's physical activity guidelines. Prev Med 2015;81:333-8.

14. Hallal PC, Andersen LB, Bull FC, et al. Global physical activity levels: surveillance progress, pitfalls, and prospects. Lancet 2012;380:247-57 http://www.sciencedirect.com/science/article/pii/ S0140673612606461.

15. Kohl HW, Craig CL, Lambert EV, et al. The pandemic of physical inactivity: global action for public health. Lancet 2012;380:294-305.

16. WHO | Global recommendations on physical activity for health. WHO http://www.who.int/dietphysicalactivity/factsheet_recommendations/ en/ (Accessed 31 May 2016).

17. Marques A, Peralta M, Martins J, et al. Few European Adults are Living a Healthy Lifestyle. Am J Health Promot 2019;33:391-8.

18. Biernat E, Tomaszewski P. Association of socio-economic and demographic factors with physical activity of males and females aged 20-69 years. Ann Agric Environ Med 2015;22:118-23.

19. Hagströmer M, Oja P, Sjöström M. The International Physical Activity Questionnaire (IPAQ): a study of concurrent and construct validity. Public Health Nutr 2006;9:755-62.

20. Bauman A, Bull F, Chey T, et al. The International Prevalence Study on Physical Activity: results from 20 countries. Int J Behav Nutr Phys Act 2009;6:21.

21. Vuori I, Lankenau B, Pratt M. Physical activity policy and program development: the experience in Finland. Public Health Rep 2004:119:331-45.

22. Weltgesundheitsorganisation, Regionalbüro für Europa. Physical activity strategy for the WHO European Region 2016 - 2025. 2016

23. Marques A, Martins J, Peralta M, et al. European adults' physical activity socio-demographic correlates: a cross-sectional study from the European Social Survey. PeerJ 2016;4:e2066.
24. Brug J, van der Ploeg HP, Loyen A, et al. Determinants of diet and physical activity (DEDIPAC): a summary of findings. Int $J$ Behav Nutr Phys Act 2017;14:150.

25. Biernat E, Piatkowska M. Zdrowotne rekomendacje Światowe Organizacji Zdrowia a rekreacyjna aktywnoć fizyczna Polaków. Med Sport 2013;4:255-64.

26. Biernat $\mathrm{E}$. Factors increasing the risk of inactivity among administrative, technical, and manual workers in Warszawa public institutions. Int J Occup Med Environ Health 2015;28:283-94.

27. Biernat E, Buchholtz S. The Regularities in Insufficient LeisureTime Physical Activity in Poland. Int J Environ Res Public Health 2016;13:798

28. Biernat E, Tomaszewski P. Socio-demographic and leisure activity determinants of physical activity of working warsaw residents aged 60 to 69 years. J Hum Kinet 2011;30.

29. Borch KB, Braaten T, Lund E, et al. Physical activity and mortality among Norwegian women - the Norwegian Women and Cancer Study. Clin Epidemiol 2011;3:229-35.

30. Borodulin K, Harald K, Jousilahti P, et al. Time trends in physical activity from 1982 to 2012 in Finland. Scand J Med Sci Sports 2016;26:93-100 http://onlinelibrary.wiley.com/doi/.

31. Brugnara L, Murillo S, Novials A, et al. Low Physical Activity and Its Association with Diabetes and Other Cardiovascular Risk Factors: A Nationwide, Population-Based Study. PLoS One 2016;11:e0160959.

32. Gerovasili V, Agaku IT, Vardavas $\mathrm{Cl}$, et al. Levels of physical activity among adults 18-64 years old in 28 European countries. Prev Med 2015;81:87-91.

33. Bauman AE, Reis RS, Sallis JF, et al. Correlates of physical activity: why are some people physically active and others not? Lancet 2012;380:258-71.

34. Kahlmeier S, Wijnhoven TM, Alpiger P, et al. National physical activity recommendations: systematic overview and analysis of the situation in European countries. BMC Public Health 2015;15:133.

35 WHO/Physical activity. http://www.who.int/mediacentre/factsheets/ fs385/en/ (Accessed 16 Apr 2017). 